\title{
An Analysis Method for Negative Feedback Amplifier Network Based on Dual-port Network Model
}

\author{
Li Gu, Li He, Yong Liu, \\ Department of Electronic Science and Technology, \\ University of Science and Technology of China, \\ Hefei 230026, China \\ gurr@ustc.edu, \{heli,liuyong,shengyj\}@ustc.edu.cn
}

\author{
Zhuang Sheng, Yang Wang \\ Department of Electronic Science and Technology, \\ University of Science and Technology of China, \\ Hefei 230026, China \\ shengyj@ustc.edu.cn,wyoicq@yahoo.cn
}

\begin{abstract}
Block diagram and dual-port network are common methods to solve negative feedback amplifier but with unsatisfied limitations and disadvantages. This paper addresses a new method based on dual-port network to solve the negative feedback amplifier, which shows much clear electrotechnical meaning. The effectiveness of the model is verified by two classical circuit problem and the parameter of the network are accurately given. The method can integrate the theory of dual-port modes into block diagram, which may deepen the insight for further research.
\end{abstract}

Keywords-Block diagram, Dual-port network, Negative feedback

\section{INTRODUCTION}

Negative feedback network is widly used in amplifier circuit, which can ameliorate the network's stability, spread its band pass, restrain a variety of factors caused by the linear and nonlinear distortion and improve its input and output performance.Negative feedback amplifier network can be divided into four configuration according to their different ways of sampling and summation. Block diagram is the usual way to study them.

Block diagram shows that feedback signal superimposes the input signal at the inport. When the feedback signal is inverse with the input signal, the net input signal will decrease, resulting in a corresponding decrease of the output signal. The feedback network $F$ of this kind is callegd negative feedback network and the amplifier network $A_{F}$ negative feedback amplifier network.

According to block diagram we can derive the overall gain: $\dot{A}_{F}=\frac{\dot{A}}{1+\dot{A} \dot{F}}$, the factor $\dot{A}$ is called the open-loop amplication; especially when the conditions meets the deep negative feedback $(|A \cdot F| \gg>1$, Which usually satisfies the conditions automatically in negative feedback amplifier networks.), $\quad \dot{A}_{F}=\frac{\dot{A}}{1+\dot{A} \dot{F}} \doteq \frac{1}{\dot{F}}$; which shows that the amplification is independent of the amplifier network $\dot{A}$, but only dependent with the feedback factor $\dot{F}$. Because the feedback network is usually constituted by resistance network, the electrical characteristics of feeback network is stable and the amplification of negative feedback amplifier network is more stable than usual amplifier network. In addition, negative feedback network $\dot{F}$ can effectively spread the band pass, reduce the distortion and change the input and output impedance values by $(1+\dot{A} \dot{F})$ times of negative feedback amplifier network $\dot{A}_{F}$ to amplifier $\dot{A}$.

The blcok diagram is a simplified model of ideal. From the model we may know $\dot{X}_{F}=\dot{F} \cdot \dot{X}_{o}$. The effect from feedback network to the input and output performance is ignored, as a result, that makes it difficult to analyze and solve the negative feedback amplifier network: fuzzy concept of open loop and closed loop, solving of input and output impendance value and so on.Dual-port network theory is usually based on blcok diagram, as the reasons given above, it can not solve negative feedback amplifier well.

In this paper, we will study negative networks with method based on dual-port network model theory, give a clear depiction and explanation of its electrotecnical meaning and verify the validity of our method by solving the factors of negative feedback amplifier networks.

\section{DUAL-PORT NETWORK MODEL}

As the amplifier network and the negative feedback network are all dual-port network, we may study them with dual-port network model. For example, we'll study negative feedback voltage in series amplifying network (Figure.1).

For simplicity, it is assumed that the feedback network is constituted by the resistance network and the signal frequency is in the intermediate frequency (i-f) of the amplifier network. Figure. 1 shows the equivalent circuit of the amplifier network. $r_{i}, r_{o}, A_{u u \infty}$ means the input and output impendence, voltage amplification coefficient $\left(R_{L}=\infty\right.$,open load) of the amplifier network $\dot{A}$. According to the configuration of feedback network, we may get the equations of the feedback network. 


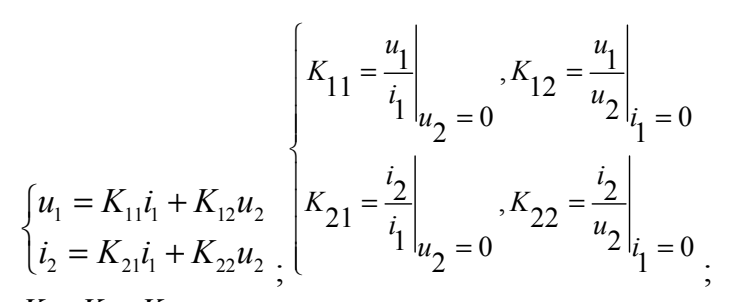

$K_{11}, K_{12}, K_{21}, K_{22}$ means the load of port.1, the voltage feedback factor, the transfer coefficient from port.1 to port.2, the load of port2. Because of one-way nature of the signal transmission, the transfer coefficient may be ignored $\left(K_{21} \doteq 0\right) . r_{1}=K_{11}, F_{u u}=K_{12}, r_{2}=K_{22}$. As Figure. 2 shows that the feedback voltage $\left(u_{f}\right)$ depends on not only the output voltage but also the load effect of feedback network. In other words, the concept "open loop" does not mean a simple removal of the feedback network but the removal of the controlled source(The feedback coefficient should be regarded as zero, $F_{u u}=0$ ).

\section{ANALYSIS AND SOLUTION}

From the above we can get the model of the negative feedback voltage in series amplifying network(Figure.3).

We may further improve the model shown in Figure. 1 and get the model shown in Figure. 4 so that the model is more in line with its electrotechnical meaning:

In Figure.4, the load effect of feedback network to the input/output port is considered as part of the amplifier network and reflected in the amplifier network. We can see the model in Figure. 4 matches the block diagram well.

According to Figure.4, We can solve the voltage gain, input and output impendence respectively:

$$
\begin{aligned}
& r_{i}^{\prime}=r_{i}+r_{1}, r_{o}^{\prime}=\frac{1}{1 / r_{o}+1 / r_{2}}, A_{u u \infty}^{\prime}=A_{u u \infty} \cdot \frac{r_{i}}{r_{i}^{\prime}}, A_{u u \infty}^{\prime \prime}=A_{u u \infty}{ }^{\prime} \cdot \frac{r_{o}^{\prime}}{r_{o}}
\end{aligned}
$$

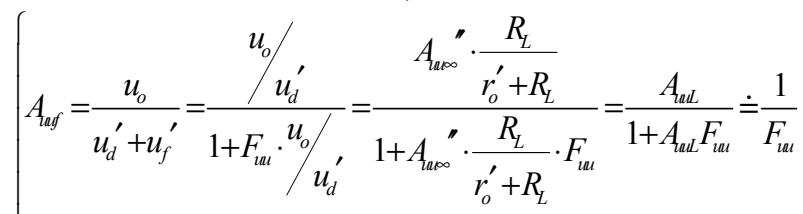

$$
\begin{aligned}
& \left\{r_{i f}=\left(1+A_{u a p \infty}{ }^{\prime \prime} F_{t u u} \frac{R_{L}}{r_{o}^{\prime}+R_{L}}\right) \cdot r_{i}^{\prime}\right. \\
& r_{o f}=\frac{r_{o}^{\prime}}{1+A_{u m \infty}^{\prime \prime} F_{u u}}
\end{aligned}
$$

$A_{u u \infty}{ }^{\prime \prime}$ is the open circuit voltage gain of open loop, $A_{u u L}$ is the open loop voltage gain considering the load resistance. Generally the overall gain $A_{u u f}$ is approximately equal to the factor $1 / F$ in the conditions of deep negative feedback. the input and output impendence changes $(1+A F)$ times than that of closed loop. For the other three configurations, we can also get the same conclusion, which readers can derive them with the method.

When there are several feedback networks $\left(\dot{F}_{1}, \dot{F}_{2}, \ldots\right)$, we may analyze the networks layer by layer. For example we can combine the feedback network $\left(F_{1}\right)$ and amplifier network $(A)$ to a whole amplifier network $\left(A^{\prime}\right)$ while the other feedback network works independently.

\section{EXAMPLES AND VERIFICATION}

After method obtained from above, we will study some negative feedback amplifier of two feedback configuration.

\section{A. 4.1 noninverting scaling operation circuit}

See the circuit given below, we'll find the feedback networkn in the pane marked with dotted lines. It is easy to judge its configuration which is negative feedback voltage in series. From the above we will easy solve the network:

$$
F_{u u}=K_{11}=\frac{R+R_{f}}{R}, r_{1}=\frac{1}{1 / R^{+} / R_{f}}, r_{2}=R+R_{f}, r_{i}^{\prime}=\infty, r_{o}^{\prime}=0, r_{i f}=\infty, r_{o f}=0 \text {. }
$$
value.

We can see the solution is identical with the theoretical

\section{B. 4.2 single stage quasi-common emitter amplifier}

See the circuit given in Figure.6, we will see the feedback configuration is current negative feedback in series: As the figure shown above, we can get the model. $R_{B}, R_{C}$ is outside of negative feedback amplifier network so that its effect may be considered at last.

According to the method given above, we firstly study the feedback network and get the factor of its open loop condition. We'll get the solution:

$$
\begin{aligned}
& \qquad r_{1}=r_{2}=R_{E}, F_{u i}=R_{E} ; \\
& r_{i}^{\prime}=r_{b e}+r_{1}=r_{b e}+R_{E}, r_{o}^{\prime}=\infty+R_{E}=\infty, A_{i u}=\left.\frac{i_{o}}{u_{i}}\right|_{F_{u i}=0}=\frac{\beta}{r_{b e}+R_{E}} \\
& A_{i u} \text { is the factor of open loop }\left(F_{u i}=0\right) \text {. Then we will obtain } \\
& \text { the factor of closed loop: }
\end{aligned}
$$

$$
\left\{\begin{array}{l}
r_{i f}=\left(1+A_{u t} \cdot F_{u i}\right) \cdot r_{i}^{\prime}=\left(1+\frac{\beta}{r_{b e}+R_{E}} \cdot R_{E}\right) \cdot\left(r_{b e}+R_{E}\right)=r_{b e}+(1+\beta) R_{E} \\
r_{o f}=\left(1+A_{u t} \cdot F_{u i}\right) \cdot r_{o}^{\prime}=\infty \\
A_{u f}=\frac{u_{o}}{u_{i}}=\frac{-i_{o} \cdot R_{L}^{\prime}}{u_{i}}=-R_{L}^{\prime} \cdot \frac{i_{o}}{u_{i}}=-R_{L}^{\prime} \cdot A_{u f}=-R_{L}^{\prime} \cdot \frac{\beta}{r_{b e}+(1+\beta) R_{E}} \doteq-R_{L}^{\prime} \cdot \frac{1}{F_{u i}}=\frac{R_{L}^{\prime}}{R_{E}} \\
R_{L}^{\prime}=\frac{1}{1 / R_{C}+1 / R_{L}}
\end{array}\right.
$$


At last, we'll get the final solution by taking into account the impact of resistance outside the loop:

$$
r_{i f}{ }^{\prime}=\frac{1}{1 / R_{B}+1 / r_{i f}}, r_{o f}{ }^{\prime}=R_{C}, A_{u u f}=-\frac{\beta R_{L}{ }^{\prime}}{r_{b e}+(1+\beta) R_{E}} \text {; }
$$

The values shown above show the effectiveness of our method.

\section{CONCLUSION}

In this paper, we analyze the structure and function of negative feedback network in negative feedback amplifier, define the concept of open loop and closed loop, make the analysis and interpretation on the various parameters with the method based on dual-port network, and get the solution of the mathematical expression. It shows that the method addressed in this paper has a very clear electrotechnical meaning and reflects the function and role of feedback networks.

By example of solving the negative feedback amplifier, the method has been proved valid and effective, and can be well unified with the block diagram of the negative feedback network.

\section{REFERENCES}

[1] Nikolic, B.; Slavoljub, M.; A general method of feedback amplifier analysis; Circuits and Systems, 1998. ISCAS '98. Proceedings of the 1998 IEEE International Symposium on Volume: 3.

[2] Cherry, E.M.; Input impedance and output impedance of feedback amplifiers Circuits, Devices and Systems, IEE Proceedings - Volume: 143 , Issue: 4 Digital Object Identifier: 10.1049/ip-cds:19960456 Publication Year: 1996, Page(s): $195-201$.

[3] Cherry, E.M.;Loop Gain, Input Impedance and Output Impedance of Feedback Amplifiers,;Circuits and Systems Magazine, IEEE, Volume: 8 , Issue: 1,2008, Page(s): 55 - 71

[4] J.L.R. Marrero, "Simplified analysis of feedback amplifiers," I.E.E.E.Trans. Education, vol. 48, pp. 53-59, Feb. 2005.

[5] E.M. Cherry, "Feedback amplifier configurations," Proc. Inst. Elec.Engrs. Pt.G, Ccts., Devices \& Systs., vol. 147-G, pp. 334-346, Dec. 2000.

[6] E.M. Cherry, "Feedback Amplifiers and the Complex Frequency Plane," book in preparation, expected completion 2008.

[7] GRAY, P.E., and SEARLE, C.L.: 'Electronic principles' (Wiley, New York, 1969), pp. 630-664.

[8] Tong Shibai, Hua Chengying, Analog Electronics(Third Edition) [M],Beijing, Higher Education Press 2001.

[9] Dai Beiqian, Linear Electronic Circuits (Second Edition), Tsinghua University Press, 2008.

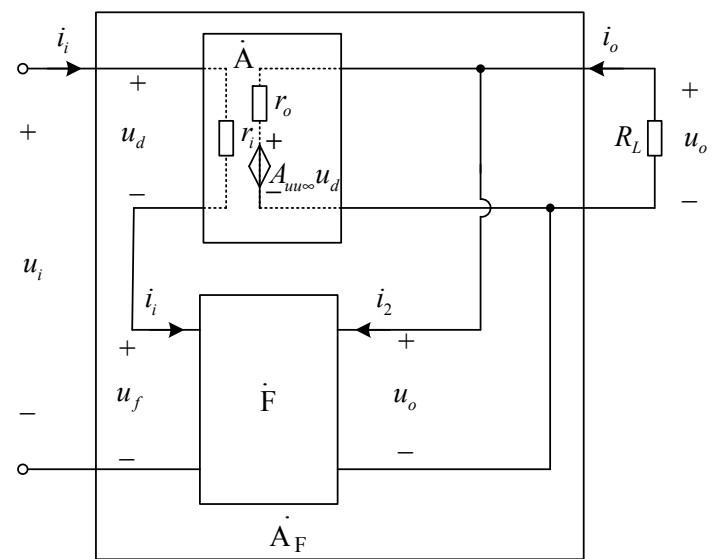

Figure1. Negative feedback voltage in series amplifying network

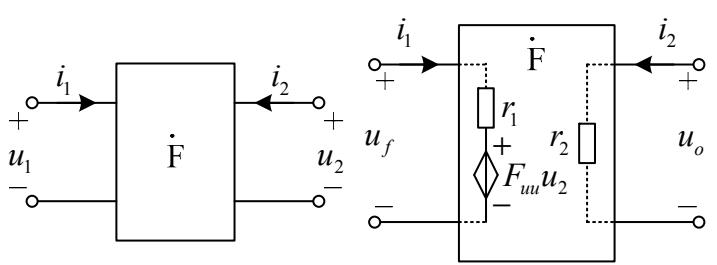

Figure 2. Dual-port model of negative feedback network 


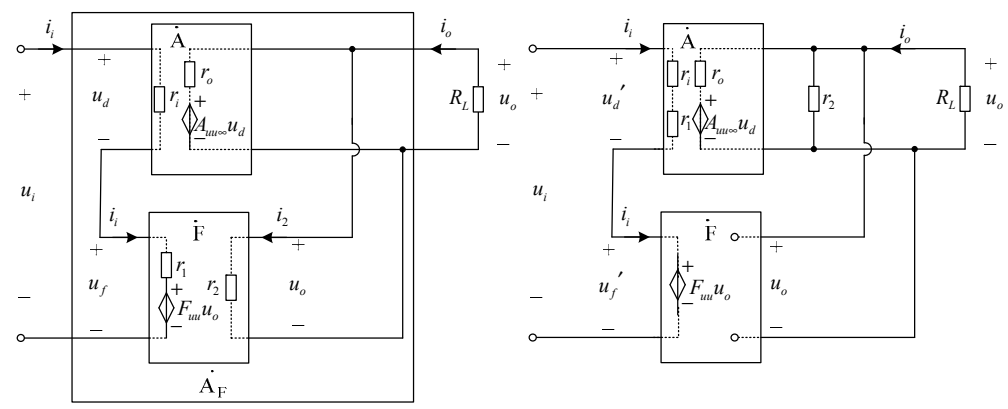

Figure. 3 Model of negative feedback voltage in series amplifying network-I

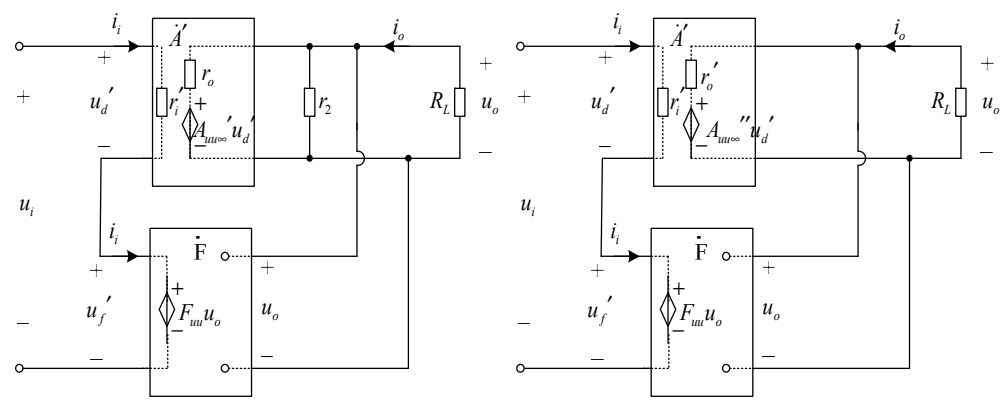

Figure 4. Model of negative feedback voltage in series amplifying network-II
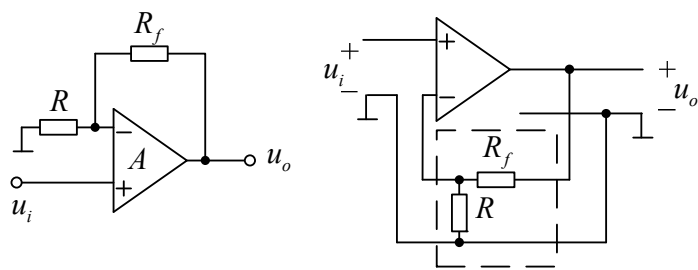

Figure 5. noninverting scaling operation circuit

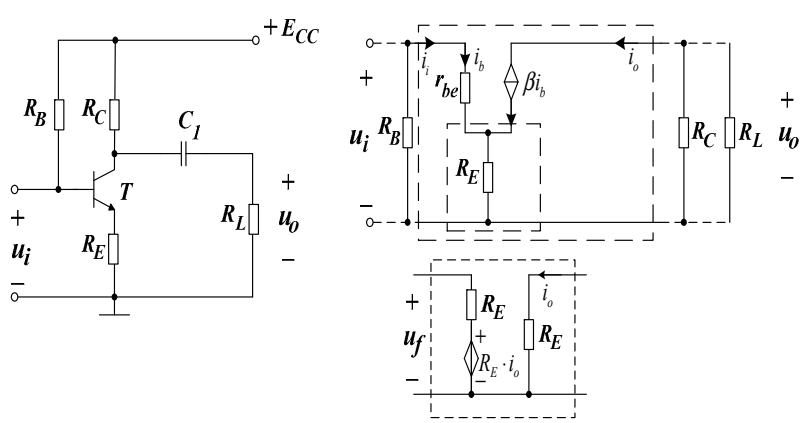

Figure 6. single stage quasi-common emitter amplifier 